\title{
A Basic Process Model of a Behavior Change Mechanism of Action
}

\author{
Martin S. Hagger ${ }^{1,2}$ \\ ${ }^{1}$ Psychological Sciences, University of California, Merced \\ ${ }^{2}$ Faculty of Sport and Health Sciences, University of Jyväskylä
}

A relatively recent contribution to knowledge of behavior change interventions has been the development of databases comprising expert-verified links between intervention content (often a behavior change technique or set of techniques) and the constructs proposed to mediate intervention effects on behavior from multiple theories (Carey et al., 2019; Connell et al., 2018; Michie et al., 2017). These databases provide detail on an important aspect of the theory-stipulated mechanisms by which behavior change interventions 'work' in changing behavior. Mechanisms also comprise links between theoretical constructs and behavior, and links between intervention content and behavior.

A basic model representing a generic mechanism of action of a behavior change intervention is illustrated in the diagram below. The upper panel of the diagram illustrates the process by which the content of a behavioral intervention (comprising one or more behavior change methods or techniques) affects change in behavioral outcomes (often a measure of behavior) through change in constructs representing the psychological process from behavioral theory. Specifically, the effect of the behavior change method or technique used in the intervention on change in a modifiable construct representing the psychological process purportedly targeted by the method or technique according to theory (known as the mediator) is illustrated (path $a$ in the upper panel of the diagram below). The effect of the theorybased construct on change in the behavioral outcome is also depicted (path $b$ in the upper panel of the diagram below). Together these effects comprise a mediated or indirect effect, which is proposed to account for the direct effect of the behavior change method or technique on behavior change (represented by path $c^{\prime}$ in the upper panel of the diagram below). If the construct fully mediates the effect of the technique on behavior change, then there will be no remaining effect of the technique on behavior change, so the size of path $c^{\prime}$ would be small or trivial in size. If there is also a non-trivial direct effect alongside the indirect effect of the intervention, known as a residual effect, then the putative 
mediator does not fully explain the process by which the intervention affects behavior change. The direct effect of the technique on behavior change independent of the mediated effect represents a test of the efficacy of the intervention content in changing behavior (represented by path $c$ in the lower panel of the diagram). The effect of the behavior change method or technique on change in behavior mediated by change in the theory-based construct can therefore be expressed as: $c-c^{\prime}=a \times b$ (for further details of these expressions, see Hayes, 2018). Together, this entire process illustrated in the diagram represents the mechanism of action (Michie et al., 2017; Rothman, Klein, \& Sheeran, 2020; Sheeran, Klein, \& Rothman, 2017; Suls et al., 2020).

It is important to note that any process evaluation of a behavior change intervention necessitates specification of such a process model a priori and the inclusion of relevant design features, measures, and data necessary to conduct the necessary mediation analysis to test the mechanism of action. Also important is that the mechanism of action illustrated likely represents a simplified form of a behavior change intervention as many behavior change methods or techniques have multiple theory-based constructs that mediate their effects on behavior - this is illustrated in the diagram by the subscripted $i$ adjacent to paths $a$ and $b$ and adjacent the mediator in the upper panel of the diagram below. Further, interventions often to adopt multiple techniques simultaneously, so without a factorial design testing the independent effects of each makes it difficult to ascertain the unique mechanisms of action.

Readers interested in further details on mechanisms of action in behavior change interventions are referred to the cited references and Hagger, Moyers, McAnally, and McKinley's (2020) recent article on the topic.

\section{References}

Carey, R. N., Connell, L. E., Johnston, M., Rothman, A. J., de Bruin, M., Kelly, M. P., \& Michie, S. (2019). Behavior change techniques and their mechanisms of action: A synthesis of links described in published intervention literature. Annals of Behavioral Medicine, 53, 693-707. https://doi.org/10.1093/abm/kay078 
Connell, L. E., Carey, R. N., de Bruin, M., Rothman, A. J., Johnston, M., Kelly, M. P., \& Michie, S. (2018). Links between behavior change techniques and mechanisms of action: An expert consensus study. Annals of Behavioral Medicine, 53, 708-720.

https://doi.org/10.1093/abm/kay082

Hagger, M. S., Moyers, S., McAnally, K., \& McKinley, L. E. (2020). Known knowns and known unknowns on behavior change interventions and mechanisms of action. Health Psychology Review. https://doi.org/10.1080/17437199.2020.1719184

Hayes, A. F. (2018). Introduction to mediation, moderation, and conditional process analysis: A regression-based approach (2nd ed.). New York, NY: Guildford Press.

Michie, S., Carey, R. N., Johnston, M., Rothman, A. J., de Bruin, M., Kelly, M. P., \& Connell, L. E. (2017). From theory-inspired to theory-based interventions: A protocol for developing and testing a methodology for linking behaviour change techniques to theoretical mechanisms of action. Annals of Behavioral Medicine, 52, 501-512. https://doi.org/10.1007/s12160-016-9816-6

Rothman, A. J., Klein, W. M. P., \& Sheeran, P. (2020). Moving from theoretical principles to intervention strategies: Applying the experimental medicine approach. In M. S. Hagger, L. D. Cameron, K. Hamilton, N. Hankonen \& T. Lintunen (Eds.), The Handbook of Behavior Change. New York, NY: Cambridge University Press.

Sheeran, P., Klein, W. M. P., \& Rothman, A. J. (2017). Health behavior change: Moving from observation to intervention. Annual Review of Psychology, 68, 573-600 https://doi.org/10.1146/annurev-psych-010416-044007

Suls, J., Mogavero, J. N., Falzon, L., Pescatello, L. S., Hennessy, E. A., \& Davidson, K. W. (2020). Health behaviour change in cardiovascular disease prevention and management: Meta-review of behavior change techniques to affect self-regulation. Health Psychology Review. https://doi.org/10.1080/17437199.2019.1691622 
Diagram of a basic model of a behavior change mechanism of action. The upper panel represents the mediation of a behavior change method or technique on behavior change through modifiable theorybased factors along with the residual effect of the technique on behavior change. The lower panel represents the direct effect of the technique on behavior change, effectively illustrating intervention efficacy in the absence of the proposed mediating theory-based factor.
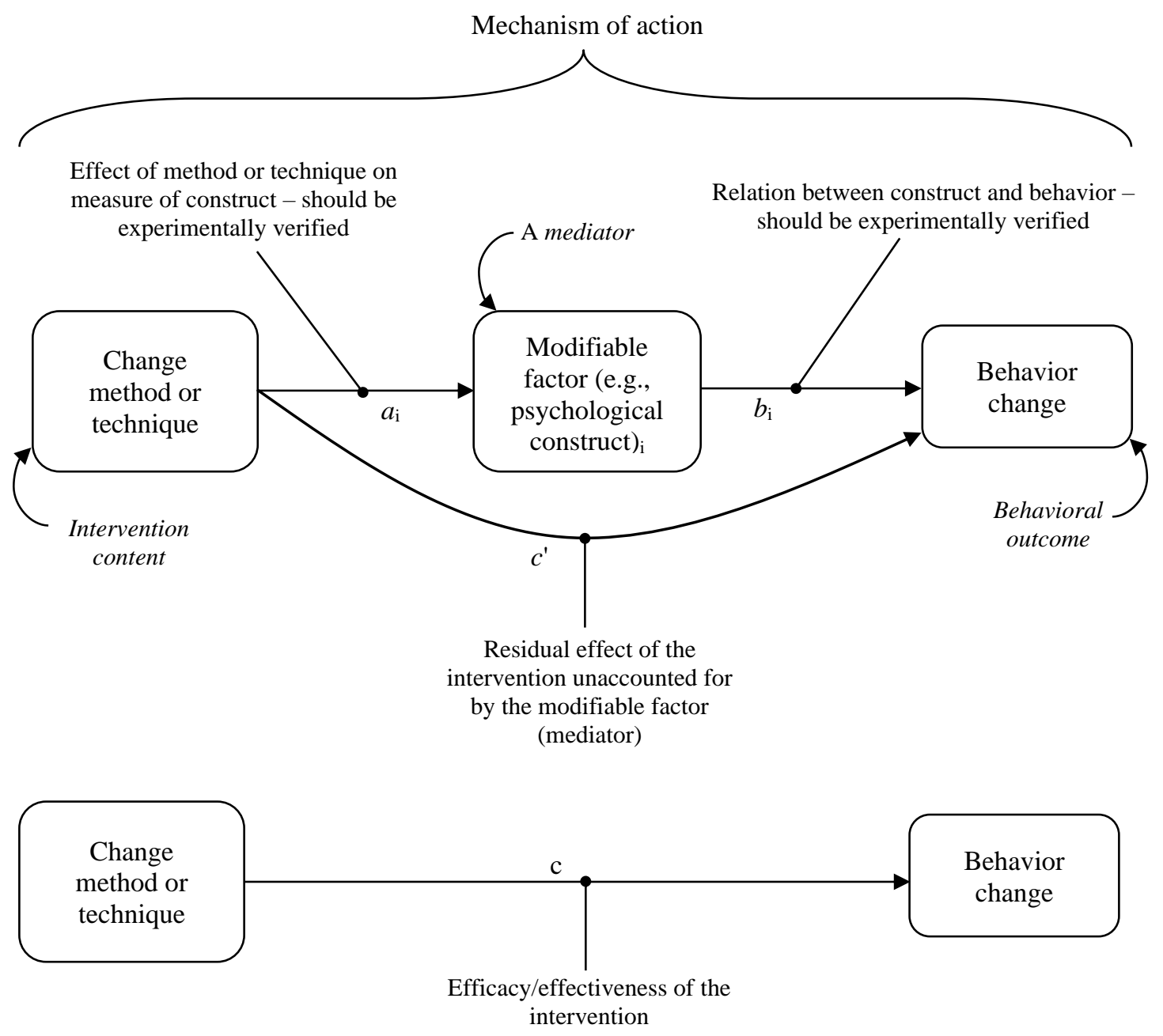